\title{
New drugs
}

\section{Aust Prescr 2019;42:168-9 https://doi.org/10.18773/ austprescr.2019.057 \\ First published \\ 13 September 2019}

\section{$<$}

Some of the views expressed in the following notes on newly approved products should be regarded as preliminary, as there may be limited published data at the time of publication, and little experience in Australia of their safety or efficacy. However, the Editorial Executive Committee believes that comments made in good faith at an early stage may still be of value. Before new drugs are prescribed, the Committee believes it is important that more detailed information is obtained from the manufacturer's approved product information, a drug information centre or some other appropriate source.

\section{Binimetinib plus encorafenib}

\section{Approved indication: metastatic melanoma \\ Binimetinib \\ Mektovi (Pierre Fabre) \\ $15 \mathrm{mg}$ film-coated tablets \\ Encorafenib \\ Braftovi (Pierre Fabre) \\ $50 \mathrm{mg}$ and $75 \mathrm{mg}$ capsules}

Many cases of melanoma involve the BRAF mutation. This results in the production of an abnormal protein kinase which promotes tumour cell growth. Drugs, such as vemurafenib and dabrafenib, that can inhibit this BRAF kinase may therefore improve survival in patients with melanoma. Another step in the pathway leading to tumour cell growth involves the MEK enzymes. These are the targets of drugs such as cobimetinib and trametinib. For patients with BRAF mutations, current treatment involves a combination of a BRAF inhibitor and a MEK inhibitor.' Encorafenib (BRAF inhibitor) and binimetinib (MEK inhibitor) are an example of such a combination for the treatment of unresectable or metastatic melanoma.

Both drugs should be swallowed whole with water. Grapefruit juice should be avoided as it interacts with encorafenib. At the recommended doses steady state is reached within 15 days. Both drugs are mainly cleared by metabolism. As the metabolism of encorafenib involves cytochrome P450 (CYP) 2C19, 2D6 and 3A4, there are many potential drug interactions. Strong inhibitors of CYP3A4 such as clarithromycin and itraconazole should be avoided. Liver disease will increase the concentrations of both drugs. A reduced dose of encorafenib is advised in mild hepatic impairment (Child Pugh A) and the combination should not be used at all with greater impairment.

The terminal half-life is about nine hours for binimetinib and six hours for encorafenib. Little active drug is excreted in the urine. No dose reductions are required in patients with mild or moderate renal impairment, but there are no data about the combination in severe impairment (<30 mL/min/1.73 $\mathrm{m}^{2}$ ).

The main study of encorafenib and binimetinib was an open-label phase III trial involving patients with a BRAF mutation and locally advanced, unresectable or metastatic melanoma. One group of 192 patients was assigned to take encorafenib 450 mg once daily with binimetinib $45 \mathrm{mg}$ twice daily. Another group of 194 was randomised to take encorafenib $300 \mathrm{mg}$ daily and a third group of 191 was assigned to take vemurafenib $960 \mathrm{mg}$ twice daily. After a median follow-up of 16.6 months, the median progression-free survival was 14.9 months with the combination. This was longer than with encorafenib alone (9.6 months) and vemurafenib (7.3 months). ${ }^{2}$

After two years, the overall survival with the combination was $57.6 \%$ compared to $49.1 \%$ with encorafenib and $43.2 \%$ with vemurafenib. At a median follow-up of 36.8 months, the median overall survival was 33.6 months with the combination, 23.5 months with encorafenib and 16.9 months with vemurafenib. ${ }^{3}$ The safety data for the trial included 570 patients. Adverse events led to $15 \%$ of the patients in the encorafenib groups and $17 \%$ of the vemurafenib group stopping treatment. Most patients required reduced doses because of adverse effects. Common adverse events that were more frequent with encorafenib plus binimetinib than with encorafenib alone included nausea, vomiting, diarrhoea, muscle spasms, hypertension, altered liver function and increased creatine kinase. Although they were common, skin reactions such as rashes were less frequent with the combination than encorafenib alone. ${ }^{3}$ Serious adverse events related to the combination include haemorrhage, left ventricular dysfunction and ocular toxicities such as retinopathy. There is a risk of new cancers such as squamous cell carcinoma.

As animal studies show fetal toxicity, the combination should not be used in pregnancy. Women should use effective contraception during treatment and for at least one month afterwards.

Although there was a significant difference in overall survival for encorafenib plus binimetinib over vemurafenib, there was no statistical advantage over encorafenib alone. ${ }^{3}$ This is despite the combination including a higher dose (450 mg) of encorafenib. The protocol of the trial has been revised so that the dose of encorafenib will be $300 \mathrm{mg}$, either alone or in the combination. This will make it easier to assess whether giving binimetinib with encorafenib adds significantly to the efficacy of encorafenib.

Other combinations of BRAF and MEK inhibitors are available and currently it is not possible to say which is the best. When the quality-of-life data for encorafenib and binimetinib are released they may assist with this choice. There are few data to show 


\section{<ustralian Prescriber}

VOLUME 42 : NUMBER 5 : OCTOBER 2019

that this combination would be effective in patients whose cancer has progressed during treatment with a different BRAF inhibitor. This combination is only approved for patients with a confirmed BRAF mutation.

$\mathbf{T}$ manufacturer provided the product information

\section{REFERENCES}

1. Atkinson V. Medical management of malignant melanoma. Aust Prescr 2015;38:74-8. https://doi.org/10.18773/ austprescr.2015.028

2. Dummer R, Ascierto PA, Gogas HJ, Arance A, Mandala M, Liszkay $\mathrm{G}$, et al. Encorafenib plus binimetinib versus vemurafenib or encorafenib in patients with BRAF-mutant melanoma (COLUMBUS): a multicentre, open-label, randomised phase 3 trial. Lancet Oncol 2018;19:603-15. https://doi.org/10.1016/S1470-2045(18)30142-6

3. Dummer R, Ascierto PA, Gogas HJ, Arance A, Mandala M, Liszkay $\mathrm{G}$, et al. Overall survival in patients with BRAFmutant melanoma receiving encorafenib plus binimetinib versus vemurafenib or encorafenib (COLUMBUS): a multicentre, open-label, randomised, phase 3 trial. Lancet Oncol 2018;19:1315-27. https://doi.org/10.1016/ S1470-2045(18)30497-2
The Transparency Score is explained in New drugs: transparency, Vol 37 No 1, Aust Prescr 2014;37:27.

At the time the comment was prepared, information about this drug was available on the websites of the Food and Drug Administration in the USA, and the European Medicines Agency. 